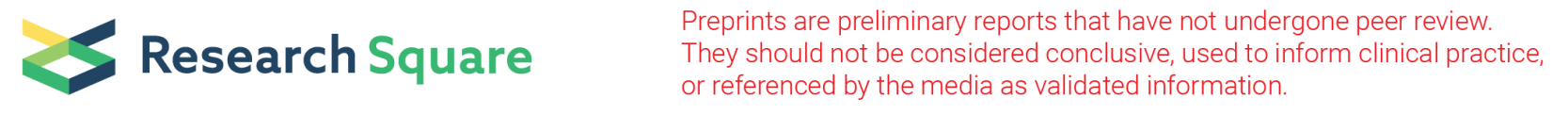

\title{
The Relationship Between the Cultural Intelligence, Communication Skills, and Social Interactions of Emergency Department Staff: A Cross-Sectional Study
}

Nasrin Hanifi ( $\square$ nasrinhanifi@gmail.com )

Zanjan University of Medical Sciences https://orcid.org/0000-0002-4027-2399

Majid Baratipour

Zanjan University of Medical Sciences

Kourosh Amini

Zanjan University of Medical Sciences

Research article

Keywords: Cultural intelligence, Communication skills, Social interactions, Emergency, Staff members, Iran

Posted Date: October 5th, 2020

DOI: https://doi.org/10.21203/rs.3.rs-74766/v1

License: (c) (i) This work is licensed under a Creative Commons Attribution 4.0 International License. Read Full License 


\section{Abstract}

Background: Globalization is accompanied by cultural diversity. Although cultural differences are an integral part of this phenomenon, it seems that language barriers would make communication difficult. This study investigates how cultural intelligence (CQ) correlates with communication skills (CSs) and social interactions (SIs) of emergency department (ED) staff members. This study investigates how cultural intelligence (CQ) correlates with communication skills (CSs) and social interactions (SIs) of the emergency department (ED) staff members.

Methods: This Cross-sectional study was performed on 197 ED staff members of three hospitals in Zanjan-Iran in 2019. The tools used for data collection include the Cultural Intelligence Scale, Communication Skills Scale, and Social Interaction Questionnaires.

Results: The results of this study revealed that the mean CQ score of participants was $85.78 \pm 6.24$ out of 140 and the total mean score of CSs was $55.41 \pm$ 3.9 out of 90 . In terms of SIs, the mean score of the positive thoughts dimension of the subjects (47.86 \pm 4.14$)$ was more than that of negative thoughts (33.01 \pm 3.92$)$. The total score of CQ had a positive correlation with CSs such that an increase in the CQ level results in an increase in positive thoughts and a decrease in negative thoughts $(P<0.001)$.

Conclusions: An increase in CQ of the ED staff members is accompanied by an increase in their CSs and positive thoughts. To improve the communication and service quality of the ED, leaders are recommended to provide some courses to enhance the CQ and cultural competence level of their employees.

\section{Background}

Culture affects the perception of health and illness, decisions for treatment, experiences, and outcomes of healthcare [1]. Cultural diversity can lead to a communication challenge in therapeutic environments and may introduce undesirable outcomes for patients [2]. When the healthcare needs of cultural diversity are not well provided, it can result in isolation and dissatisfaction of the patients. Hence, to provide effective care to patients with different cultures, it is necessary to understand their cultural diversity [3]. When cultural care is provided based on the values and beliefs of the patients, they follow the healthcare instructions more than before [4].

Cultural intelligence (CQ) of the healthcare staff members is considered among the most important and effective factors in having effective interactions with patients and their families of different cultures $[5,6]$. CQ is defined as the ability and capability of individuals to have effective performance in different cultural environments [7]. CQ is an ability directly associated with communicational skills and social interactions of the individuals. Besides, CQ eliminates the gap in the transfer of concepts from one culture to another [8]. As a result, an increase in CQ can lead to an increase in the performance and organizational commitment of staff, a decrease in their stress level, and an increase in the quality of their healthcare and organization dynamics [9]. According to previous studies, high CQ results in a decrease in the stress and anxiety of the patients and their families, thereby promoting their satisfaction [10].

The emergency department (ED) is the first medical environment that most patients experience. The healthcare staff of the ED communicates regularly with patients from different cultures [11]. In an ED, in addition to other medical treatment units, social interactions are considered among the main pillars of health care that affect the performance of staff members [12]. Some studies have reported high conflict between the patients or their families and healthcare staff members in ED. It has been evidenced that other factors such as the high-risk situation of patients and their psychological conditions that create these conflicts, the cultural diversity between staff members and patients can exacerbate these conflicts as well [13, 14]. In this context, providing high-quality service and satisfying the patients in an ED is not possible unless to understand their problems through proper communication [15]. Communication is not simply talking one to another but to understand true cultural diversity and how those differences affect both the delivery of and receiving health care. Cultural diversity, particularly not having a common language, pose barriers to having effective communication. Even nonverbal communications have various implications in different cultures [16]. Proper knowledge of clinicians about the culture, language, values, traditions, practices, and beliefs of patients can be of great assistance in communicating with patients without prejudgment [17]. In this regard, the communication and interactions of ED staff members are of particular importance in the satisfaction of patients and their relatives $[18,19]$.

Therefore, studying the status of communication skills (CSs) and social interactions (SIs) in healthcare staff members in multicultural environments may have great benefits. Since patients and their families face different workers in the ED, the proper interaction between them is critical. Considering the multicultural nature of the city of Zanjan and the lack of a study in this regard, this study aimed to investigate the status of cultural intelligence and its relationship with social interactions and communication skills of staff members in the ED of hospitals affiliated to Zanjan University of Medical Sciences.

\section{Methods}

\section{Study design and sampling}

The statistical population of the present cross-sectional study included all staff members of the ED working in three hospitals (Ayatollah Mousavi, Vali Asr and Beheshti hospitals) affiliated to Zanjan University of Medical Sciences in Iran. The ED of these three hospitals have 158 active beds in the medical, trauma, cardiovascular, pediatrics, phycology, and midwifery units and 287 staff members. These members include 149 nurses with MSc, BS, and diploma education, 40 practical nurses, 30 housekeepers, 23 ED guards, 26 general physicians, and 19 emergency physicians. The other medical experts such as cardiologists, gynecologists, pediatrics, psychiatrist, and surgeons were not included in this study due to their irregular attendance in the ED.

In Iranian hospitals, the patients first are referred to the triage unit nurse and then to the general physician. If the patient must be hospitalized, he/she is visited and triaged by the emergency physician. Based on the initial diagnosis of the emergency physician, the patient is referred to different medical, or surgery units. Patient care in the ED is performed by nurses with the education levels of master of sciences of emergency nursing (MSEN), bachelor of science of nursing (BSN), and diploma of nursing (DN). Primary health care of the patients is provided by licensed practical nurses. Although practical nurses do not have 
specialized nursing education, they are trained through short courses. Cleaning the units and patients transfer to other units is performed by housekeepers. To prevent unnecessary referral of patients' families to the ED and prevent probable conflicts between the staff members and patients' families, there are at least three guards at the entrance of the ED. Practical nurses, guards, and housekeepers have different levels of education ranging from diploma to even academic education. Some of these highly educated people work in such jobs due to not finding a proper job related to their field of study.

In the present research, the sample size was estimated to be 197 by considering a $95 \%$ confidence level, 0.1 effect size and $80 \%$ power. The inclusion criteria of the study were having at least a one-year job experience in the ED and the willingness to participate in the study. Finally, 217 questionnaires were distributed among the eligible ED staff members. The inclusion criteria were: (1) at least one year of experience working in the ED and (2) the desire to participate in the study.

\section{Study setting}

Iran has 24 cultural and religious ethnicities [20]. The official language of the country is Persian and its official state religion is (Shia) Muslim. The Zanjan Province with a population of $1,057,461$ and 8 cities is located in the northwest of Iran. The capital of this province is Zanjan city. The urbanization rate in Zanjan province over the past 40 years has jumped from $28.8 \%$ to $63.4 \%$. Moreover, migration from other cities has caused a variety of demographic and cultural diversities. The population of Zanjan speaks the Azari language and their religion is (Shia) Muslim. Due to the proximity to the Kurdish cities and the northern cities of Iran, where the people speak in local dialects, the city encounters a wide variety of languages (Gilaki dialect and Kurdish language) and (Sunni) Muslim. The location of Zanjan city across the Silk Road has created many job opportunities since many decades ago. Moreover, the city has several mines that have attracted many immigrants from other cities. Today, some immigrants from other countries such as China, Afghanistan, and Turkey live in Zanjan city. Moreover, because the city is located in the Iran transit route to Europe, the staff members of the ED, visit foreign victims of traffic accidents, as well. Some ED staff members of the city are non-native and speak only Persian. On the other hand, most illiterate patients cannot speak Persian and they only speak their native language and dialect. Thus, communication between staff and patients difficult is a difficult task.

\section{Data collection}

The data needed in the present research were collected using three questionnaires of the Cultural Intelligence Scale (CQS), Social Interaction Questionnaire (SIQ), and Communication Skills Scale (CSS). All three scales were translated to Persian and back-translated to English. Next, all three retranslated scales were matched to their original version by a person proficient in English. In our study, qualitative and quantitative content validity were used to assess the validity of the scales. The scales were provided to 10 experts and the necessary corrections were made. Finally, the content validity index and content validity ratio of the scales were calculated.

The researcher referred to the ED and explained the study goals to the people meeting the inclusion criteria. Then, if they were willing to participate in the study, they submitted informed consent and received a small gift for completing the questionnaire. The questionnaires were distributed on paper. The time of submitting the questionnaire to the researcher was decided by the participants such that they could fill it accurately and conveniently. The deadline for completing the questionnaires was two weeks. Data were collected over a period of three months.

\section{Data gathering tools}

\section{CQS}

CQ was evaluated using the CQS [21]. The scale includes 4 dimensions and 20 items (metacognitive CQ, 4 items; cognitive CQ, 6 items; motivational CQ, 5 items; and behavioral CQ, 5 items). It is scored by a 7-point scale (ranging from 1 = strongly disagree to 7 = strongly agree), with its scores varying from 20 to 140 (20-60 = Low CQ; 61-100 = Moderate CQ; and 101-140 = High CQ). This scale has been used in several studies in Iran [22, 23]. Shomoossi et al. (2019) in a study on 136 employees in Sabzevar University of Medical Sciences calculated the reliability of the total score of CQ using Cronbach's alpha as 0.85 [23], while in our study it was 0.75 . In this study, the content validity index and content validity ratio were estimated to be 0.83 and 0.79 , respectively.

\section{$S I Q$}

To study the SIs of the participants, the 30-item Social Interaction Self-Statement Test (SISST) was used [24]. This scale includes two dimensions of negative thoughts (NTs) and positive thoughts (PTs) in communication. Each dimension contains a 15-item 5-point Likert scale, where the scores vary from 15 to 75 . A high score in the negative dimension indicates the weak Sls, suggesting that the individuals believe in the negative role of inappropriate conditions in communicating and feeling fear and anger in social situations. However, a high score in the positive dimension shows the high ability of individuals in communicating with others, low anxiety, and their belief in facilitating communication with others. In the present study, the reliability of the questionnaire was calculated to be $a=0.701$ by Cronbach's alpha. Also, its content validity index and content validity ratio were estimated to be 0.87 and 0.89 , respectively.

\section{CSs}

CSs were calculated using an 18-item scale [25]. The scale has three dimensions of verbal, listening, and feedback skills, each containing 6 items. The scoring is done based on a 5-point Likert scale. The minimum and maximum scores of each dimension are 6 and 30 , respectively. The total score of the scale varies from 18 to 90 ( $<42=$ Low CS; $42-66=$ Moderate CS; and >66 = High CS). In Iran, the scale was psychometrically analyzed on 191 health volunteers and its Cronbach's alpha was reported to be $0.91^{29}$; however, in our study, the reliability of the scale was 0.787 . Also, in this study, the content validity index and content validity ratio was estimated to be 0.87 and 0.89 , respectively.

\section{Ethical considerations}


This study was conducted after granting the approval of the ethics committee of Zanjan University of Medical Sciences and obtaining an ethical code (IR.ZUMS.REC.1396.305). The researcher, after referring to the research environment and explaining the research goals, received written consent from the participants. The participants were assured that all their information would be kept confidential and they could leave the study any time they want. To make the participant comfortable and control the confounding factors such as noise and workload in the hospital, they were asked to complete the questionnaires at any time they desire.

\section{Data analysis}

The data were analyzed using SPSS V.16 software. The Kolmogorov-Smirnov test was applied to evaluate the normality of the data. The results showed that the data had a normal distribution. Independent t-test and analysis of variance (ANOVA) test were performed to evaluate the relationship between the demographic variables and three main variables. Moreover, the Pearson correlation coefficient was applied to investigate the relationship of CQ with communication skills and social interactions. Finally, the General Linear Multivariate Model test was applied to predict the CQ for criterion variables including communication skills and the positive and negative thought dimensions of the social interaction questionnaire.

\section{Results}

Of the 217 questionnaires distributed, 200 questionnaires were filled by the staff members and returned to the researcher. Among the filled questionnaires, only three ones were excluded from the samples due to missing data. Finally, the information on 197 subjects was analyzed. Most of the participants were female and nurses with different educational levels. The demographic information of the participants is presented in Table 1.

Table 1

Demographic variables of the participants

\begin{tabular}{|lll|}
\hline Demographic variable & Number & Percentage \\
\hline Sex & & \\
\hline Male & 94 & 47.7 \\
\hline Female & 103 & 52.3 \\
\hline Marital status & & \\
\hline Single & 66 & 33.5 \\
\hline Married & 131 & 66.5 \\
\hline Age & & \\
\hline Less than 25 years old & 26 & 13.2 \\
\hline 25-34 years old & 68 & 34.5 \\
\hline 35-44 years old & 55 & 27.9 \\
\hline 45-54 years old & 37 & 18.8 \\
\hline More than 54 years old & 11 & 5.6 \\
\hline Occupational type & & \\
\hline Housekeepers & 18 & 9.1 \\
\hline Emergency department guard & 16 & 8.1 \\
\hline Nurse aid & 24 & 12.2 \\
\hline Nurse (Diploma) & 42 & 21.3 \\
\hline Nurse (Bachelor and master) & 85 & 43.1 \\
\hline Doctor & 12 & 6.1 \\
\hline Education & 32 & 12.2 \\
\hline Diploma & 91 & \\
\hline Associate & & 19.3 \\
\hline Bachelor & & \\
\hline Master & 36.2 \\
\hline Doctor of Medicine & 12 & \\
\hline
\end{tabular}

The findings of this study indicated that the mean CQ of the participants was $85.78 \pm 6.26$ of 140 so that $99.5 \%$ ( 196 subjects) and $0.5 \%$ of the staff members had moderate (61-100) and high CQ levels (101-140), respectively. In terms of Sls, the mean scores of PTs dimension (47.86 \pm 4.14$)$ were more than the 
mean scores of NTs. Moreover, CSs total score was $55.41 \pm 3.9$ out of 90 so that $100 \%$ (197 subjects) of the participants had moderate CSs (43 to 66 ) (Table 2).

Table 2

The mean score of CQ, SIQ, and CSS in the participants

\begin{tabular}{|llll|}
\hline Variable & Minimum & Maximum & Mean \pm SD \\
\hline CQ & & & \\
\hline Metacognitive & 10 & 22 & $16.06 \pm 2.06$ \\
\hline Cognitive & 17 & 34 & $25.98 \pm 3.1$ \\
\hline Motivational & 15 & 27 & $21.31 \pm 2.22$ \\
\hline Behavioral & 17 & 29 & $22.59 \pm 2.21$ \\
\hline Total score of dimensions & 69 & 102 & $85.78 \pm 6.24$ \\
\hline SIQ & & & \\
\hline PTs & 36 & 58 & $47.86 \pm 4.41$ \\
\hline NTs & 22 & 43 & $33.01 \pm 3.92$ \\
\hline CSS & & & \\
\hline Verbal skills & 14 & 27 & $19.71 \pm 2.12$ \\
\hline Aural skills & 10 & 23 & $17.44 \pm 2.29$ \\
\hline Feedback Skills & 12 & 24 & $18.25 \pm 2.05$ \\
\hline Total score of dimensions & 45 & 66 & $55.41 \pm 3.09$ \\
\hline
\end{tabular}

The results of the t-test showed that sex and marital status did not correlate with CQ, PTs, NTs, and CSs. Also, the ANOVA results demonstrated that occupation type and education level did not correlate with CQ, PTs, NTs, and CSs (Table 3). 
Table 3

The relationship between demographic variables with

$\mathrm{CQ}$, Sls, and CSS in the participants

\begin{tabular}{|llll|}
\hline Demographic variable & df & t/F & P value \\
\hline CQ & & & \\
\hline Mex & 195 & 0.331 & 0.093 \\
\hline Age & 195 & 0.541 & 0.654 \\
\hline Occupation type & 4 & 2.25 & 0.065 \\
\hline Education & 5 & 1.25 & 0.28 \\
\hline Sls & 4 & 0.75 & 0.56 \\
\hline PTs & & & \\
\hline Sex & & & \\
\hline Marital status & 195 & 1.7 & 0.79 \\
\hline Age & 4 & 1.8 & 0.13 \\
\hline Occupation type & 5 & 1.3 & 0.27 \\
\hline Education & 195 & 1.7 & 0.79 \\
\hline NTs & & & \\
\hline Sex & 195 & -0.56 & 0.301 \\
\hline Marital status & 195 & 1.18 & 0.197 \\
\hline Age & 4 & 1.64 & 0.164 \\
\hline Occupation type & 5 & 0913 & 0.47 \\
\hline Education & 4 & 1.3 & 0.77 \\
\hline CSS & & & 0.77 \\
\hline Sex & 195 & 0.273 & 0.519 \\
\hline Marital status & & & 0.835 \\
\hline Age & & & \\
\hline Occupation type & & & \\
\hline Education & & & \\
\hline
\end{tabular}

The results of the Pearson correlation coefficient test indicated the statistically significant and positive relationship between the total score of CQ and CSs. Moreover, a significant positive relationship was observed between verbal skills, Metacognitive CQ, and Cognitive CQ. There was a statistically significant positive relationship between listening skills with Cognitive CQ and Motivational CQ dimensions. Based on the obtained results, only the feedback dimension had a positive and significant relationship with the dimension of behavioral CQ (Table 4). 
Table 4

The relationship of CQ, SIQ, and CSS in the participants

\begin{tabular}{|c|c|c|c|c|c|c|c|c|c|c|c|}
\hline \multirow[t]{3}{*}{ Variable } & $\mathrm{CQ} /$ & $\mathrm{CQ} /$ & $\mathrm{CQ} /$ & $\mathrm{CQ} /$ & $\mathrm{CQ} /$ & CSS/ & CSS/ & CSS/ & CSS/ & $\begin{array}{l}\text { SIQ/ } \\
\text { PTs }\end{array}$ & $\begin{array}{l}\text { SIQ/ } \\
\text { NTS }\end{array}$ \\
\hline & Metacognitive & Cognitive & Motivational & Behavioral & $\begin{array}{l}\text { The total } \\
\text { score of } \\
\text { dimensions }\end{array}$ & $\begin{array}{l}\text { Verbal } \\
\text { skills }\end{array}$ & $\begin{array}{l}\text { Aural } \\
\text { skills }\end{array}$ & $\begin{array}{l}\text { Feedback } \\
\text { Skills }\end{array}$ & $\begin{array}{l}\text { The total } \\
\text { score of } \\
\text { dimensions }\end{array}$ & & \\
\hline & $\mathbf{r}$ & $\mathbf{r}$ & $\mathbf{r}$ & $\mathbf{r}$ & $\mathbf{r}$ & $\mathbf{r}$ & $\mathbf{r}$ & $\mathbf{r}$ & $\mathbf{r}$ & $\mathbf{r}$ & $\mathbf{r}$ \\
\hline
\end{tabular}

CQ

Metacognitive 1

$\begin{array}{lll}\text { Cognitive } & .235^{\star *} & 1\end{array}$

$\begin{array}{llllll}\text { Motivational } & .166^{\star} & .243^{\star *} & 1 & & \\ \text { Behavioral } & .272^{\star *} & .201^{\star *} & .255^{\star \star} & 1 & \\ \begin{array}{l}\text { Total score of } \\ \text { dimensions }\end{array} & .608^{\star *} & .736^{\star *} & .619^{\star *} & .621^{\star *} & 1\end{array}$

cSS

\begin{tabular}{|c|c|c|c|c|c|c|c|c|c|c|c|}
\hline Verbal skills & $.169 *$ & $.228^{\star \star}$ & .092 & .103 & .103 & 1 & & & & & \\
\hline Aural skills & .067 & $.158^{\star}$ & $.208^{*}$ & .14 & $.223^{\star \star}$ & .067 & 1 & & & & \\
\hline $\begin{array}{l}\text { Feedback } \\
\text { Skills }\end{array}$ & .119 & .054 & .117 & $.244^{\star \star}$ & $.191^{\star \star}$ & -.032 & .088 & 1 & & & \\
\hline $\begin{array}{l}\text { Total score of } \\
\text { dimensions }\end{array}$ & $.194^{\star *}$ & $.246^{\star \star}$ & $.235^{\star \star}$ & $.267^{\star \star}$ & $.361^{\star \star}$ & $.567^{\star \star}$ & .627 ** & $.561^{\star \star}$ & 1 & & \\
\hline \multicolumn{12}{|l|}{ SIQ } \\
\hline PTs & $.172^{\star}$ & $.143^{\star}$ & $.24^{\star \star}$ & $.279 * \star$ & $.308^{\star \star}$ & $.149 *$ & .112 & .021 & $.158^{\star}$ & 1 & \\
\hline NTs & -.056 & $-.153^{\star}$ & $-.156^{\star}$ & $-.198^{\star \star}$ & $-.218^{\star *}$ & -.058 & -.06 & $-.174^{\star}$ & $-.159 *$ & -.116 & 1 \\
\hline
\end{tabular}

*P-value $<.05$

**P-value $<.001$

The results of the Pearson correlation coefficient test revealed the significant and positive relationship between the PTs dimensions of SIQ, CQ total score, and all dimensions of CQ so that an increase in CQ level correlates with an increase in PTs. Moreover, there was a statistically significant negative relationship between the NTs dimension of social interactions scale, CQ total score, and all dimensions of CQ, except the metacognitive CQ dimension that correlates with a decrease in NTs (Table 4).

The result of the Pearson correlation coefficient test indicated a statistically significant positive relationship between the PTs dimension of social interactions scale with verbal skills and total score of CSs so that an increase in CSs is associated with an increase in PTs. Moreover, there was a significant negative relationship between the NTs dimension of SIQ, feedback skills, and total score of CSs such that an increase in the total score of CSs correlates with a decrease in NTs (Table 4). The results of the general linear model show that CQ correlates with PTs (Table 5).

Table 5

The relationship of CQ with PTs, NTs, and CSS in the participants using the General Linear Model

\begin{tabular}{|llllllll|}
\hline Criterion Variable & Type III Sum of Squares & df & Mean Square & F & P-value & R Squared & Adjusted R Squared \\
\hline PTs & 14.763 & 30 & 29.186 & .859 & .005 & .197 & .052 \\
\hline NTs & 33.746 & 30 & 19.849 & 2.201 & .117 & .26 & .12 \\
\hline CSS & 171.321 & 30 & 82.590 & 2.840 & .075 & .208 & .064 \\
\hline
\end{tabular}

\section{Discussion}

The findings of the present research revealed that the CQ level and CSs of the staff members in the ED were at a moderate level. Also, in terms of SIQ, the scores of PTs were higher than NTs. CQ had a statistically significant positive correlation with CSs in participants. Based on the obtained results, an increase in the CQ level correlates with an increase in PTs and a decrease in NTs.

In agreement with the results of our study, another work showed that the CQ of the staff members were at a moderate level [26]. Also, some studies indicated a low communication level between medical staff members and patients [2, 16, 27]. However, it seems that staff members in EDs require higher CQ and CSs.

Cultural intelligence plays an important role in staff members who daily deal with different people with different cultures. Considering the need for establishing communication between staff members and patients to prevent undesirable outcomes, cultural diversity should not hinder the proper 
communication among staff members and patients. In line with the results of the present research, Buker et al. (2014) demonstrated the positive and significant relationship between $\mathrm{CQ}$ and the effectiveness of communication [28].

In the present research, CQ was positively associated with PTs and negatively associated with NTs of SIQ. Since some parts of the SIs and CSs are associated with non-verbal communication skills, it seems that in our study, staff members with higher CQ levels have higher non-verbal skills and their language barrier can be solved in this way.

To have effective communication in the hospitals, it is necessary to enhance the CQ and cultural competence of staff because of the changes in the cultural diversity [29, 30]. High CQ and cultural sensitivity of the staff members not only improve the relationship among patients and staff members but also enhance their performance. As a result, it reduces the stress level of staff members working with patients with different cultures [31]. The results of a study showed that metacognitive CQ and cognitive CQ, motivational CQ and behavioral CQ, and metacognitive CQ and behavioral CQ could predict the cultural judgment of the staff members, cultural adaptation, and task performance, respectively [8]. In contrast with the results of our research, Ahanchian et al. (2012) reported the lack of any significant relationship between CQ and Sis [26]. The difference between our study and that of Ahanachian et al. (2012) could be attributed to the communication of staff members of the ED with different people because of the nature of the unit. Ahnachian et al. (2012) selected the samples from all general units. In comparison, in our study, the participants had at least two years of work experience in the ED; therefore, contacting more patients in the ED may result in an increase in CQ and PTs in Sls of emergency staff members. In the ED, patients and their families are more sensitive. Under such stressful conditions, cultural characteristics and differences are more obvious. Hence, better and quicker perception and analysis of proper behaviors from different cultures correlates with patients' satisfaction. Typically, managers tend to assign employees with effective CSs to the EDs. However, they may forget that the stress-resolving skills of such staff members are because of their high cultural intelligence. By understating various cultural contexts, such employees offer peace to the ED. In general units, communications occur more peacefully and the staff members have enough time to analyze the behavior and understand the cultural contexts of the referents. Besides, since patients and their families experience less stress, cultural diversity between the patients and staff members are less apparent under such conditions.

It has been reported that frequent contact with different cultures associates with an increase in cultural competence of staff members [11, 32, 33]. Furthermore, it has been evidenced that training the cultural competence and practicing cultural competency communication increase the CSs between staff members and students [34-37]. Therefore, authorities are suggested to hold education workshops on dealing with different cultures such that to promote the cultural intelligence and competence of ED staff members. In this way, they can improve communication and interactions of staff members with patients and their families.

\section{Limitation}

In Iran, despite considerable cultural diversity, few studies have been conducted on CQ. The present study aimed to provide basic knowledge about the state of CQ, CSs, and SIs of ED staff members in Zanjan City. However, the obtained results are not generalizable to other societies due to the specific culture of Zanjan. Moreover, since all questionnaires were simultaneously provided to the participants, social response bias may threaten the validity of the results. Thus, the researchers tried to minimize this bias by anonymizing the name of those who filled the questionnaires.

\section{Conclusion}

According to the results of the present study, an increase in the CQ level associated with an increase in CSs and SIs of the ED staff members. Considering the globalization and facing different cultures and ethnicities, the managers should increase the CQ level of the staff members. In this way, they can enhance their cultural competence to increase their service quality and patients' satisfaction level. Since most parts of the CQ, CSs, and SIs are acquisitive and they can be enhanced, authorities of the medical science education should try to increase CQ and cultural competence of students in a multiethnic country such as Iran to have healthcare staff members with high $\mathrm{CQ}$ in the future. Also, hospital managers should identify the strengths and weaknesses of their staff members by measuring their $\mathrm{CQ}$ and provide retraining courses based on the obtained results.

\section{Abbreviations}

CQ

Cultural intelligence; CSs:Communication skills; SIs:Social interactions; ED:Emergency department

\section{Declarations}

\section{Ethics approval and consent to participate}

This research was conducted after granting the approval of the ethics committee of Zanjan University of Medical Sciences and obtaining an ethical code (IR.ZUMS.REC.1396.305).

\section{Consent for publication}

Informed consent was obtained from all participants in the form of written.

\section{Availability of data and materials}

Not applicable 


\section{Competing interests}

The authors declare that they have no competing interests.

\section{Funding}

This article is the result of Master of Nursing thesis which funded by research department of Zanjan University of Medical Sciences.

\section{Authors' contributions}

$\mathrm{NH}$ designed the study, carried out statistical analyses of the data, was involved in the interpretation of the data and wrote the manuscript, MB collected the data, was involved in the interpretation of the data, KA was involved in the interpretation of the data. All authors read and approved the final manuscript.

\section{Acknowledgements}

This article is taken from the master's thesis in Emergency Nursing of the first author with A-12-148-13 code. We would like to show our gratitude to the research deputy of Zanjan University of Medical Sciences because of their financial supports and the sincere cooperation of the staffs in the emergency departments of Zanjan hospitals.

\section{References}

1. Goren H, Yemini M: Global citizenship education redefined-A systematic review of empirical studies on global citizenship education. Int J Educ Res. 2017, 82:170-183.

2. Foronda C, MacWilliams B, McArthur E: Interprofessional communication in healthcare: An integrative review. Nurse Educ Pract. 2016, 19:36-40.

3. Schinkel S, Schouten BC, Street Jr RL, van den Putte B, van Weert JC: Enhancing health communication outcomes among ethnic minority patients: the effects of the match between participation preferences and perceptions and doctor-patient concordance. J Health Commun .2016, 21(12):1251-1259.

4. Shen Z: Cultural competence models and cultural competence assessment instruments in nursing: a literature review. J Transcult Nurs. 2015, 26(3):308321.

5. Alizadeh S, Chavan M: Cultural competence dimensions and outcomes: a systematic review of the literature. Health Soc Care Community. 2016, 24(6):e117-e130.

6. Edwards JB: Cultural intelligence for clinical social work practice. Clin Soc Work J. 2016, 44(3):211-220.

7. Ott DL, Michailova S: Cultural intelligence: A review and new research avenues. Int J Manage Rev. 2018, 20(1):99-119.

8. Ang S, Van Dyne L, Koh C, Ng KY, Templer KJ, Tay C, Chandrasekar NA: Cultural intelligence: Its measurement and effects on cultural judgment and decision making, cultural adaptation and task performance. Manage and organ rev. 2007, 3(3):335-371.

9. Jyoti J, Kour S: Factors affecting cultural intelligence and its impact on job performance: Role of cross-cultural adjustment, experience and perceived social support. Personnel Rev. 2017, 46(4):767-791.

10. Curtis K, Wiseman T: Back to basics-Essential nursing care in the ED: Part One. Australas Emerg Nurs J. 2008, 11(1):49-53.

11. Roh H, Nirta L: Medical students interact with multicultural patients to learn cultural diversity. Korean J Med Educ 2018, $30(2): 161$.

12. Song HS, Choi J, Son YJ: The relationship between professional communication competences and nursing performance of critical care nurses in $\mathrm{S}$ outh $\mathrm{K}$ orea. Int J Nurs Pract. 2017, 23(5):e12576.

13. Repo H, Vahlberg T, Salminen L, Papadopoulos I, Leino-Kilpi H: The cultural competence of graduating nursing students. J Transcult Nurs. 2017, 28(1):98107.

14. Copeland D, Henry M: Workplace violence and perceptions of safety among emergency department staff members: Experiences, expectations, tolerance, reporting, and recommendations. J Trauma Nurs. 2017, 24(2):65-77.

15. Hettinger AZ, Benda N, Roth E, Hoffman D, lyer A, Franklin E, Perry S, Fairbanks R, Bisantz AM: Ten Best Practices for Improving Emergency Medicine Provider-Nurse Communication. J Emerg Med. 2020.

16. Norouzinia R, Aghabarari M, Shiri M, Karimi M, Samami E: Communication barriers perceived by nurses and patients. Glob Health Sci Pract. $2016,8(6): 65$.

17. Brown EA, Bekker HL, Davison SN, Koffman J, Schell JO: Supportive care: Communication strategies to improve cultural competence in shared decision making. Clin J Am Soc Nephrol. 2016, 11(10):1902-1908.

18. Forsgärde E-S, Attebring MF, Elmqvist C: Powerlessness: Dissatisfied patients' and relatives' experience of their emergency department visit. Int Emerg Nurs. 2016, 25:32-36.

19. Roh H, Park KH: A scoping review: communication between emergency physicians and patients in the emergency department. J Emerg Med. 2016, 50(5):734-743.

20. Soltanizadeh N, Alaei S: The role of culture economics in social health: the case of cultural diversity in Iran. Soc Determin Health. 2016, 2(3):115-121.

21. Earley PC, Ang S: Cultural intelligence: Individual interactions across cultures: Stanford University Press; 2003.

22. Rahimaghaee F, Mozdbar R: Cultural intelligence and its relation with professional competency in nurses. Nurs Pract Today. 2017, 4(3):115-124.

23. Shomoossi N, Asor AA, Kooshan M, Rad M: Interculturality and cultural intelligence in an academic context: A report from university staff interacting with nursing students. J Educ Health Promot. 2019, 8. 
24. Glass CR, Merluzzi TV, Biever JL, Larsen KH: Cognitive assessment of social anxiety: Development and validation of a self-statement questionnaire. Cognit Therap Res. 1982, 6(1):37-55.

25. Ersanlı K, Balcı S: Developing a communication skill inventory: Its validity and reliability. Turk Psychol Couns and Guidence J .1998, 10(2):7-12.

26. Ahanchian MR, Amiri R, Bakhshi M: Correlation between cultural intelligence and social interaction of nurses. J Health Promot Manage. 2012 , 1(2):44-53.

27. McCarthy DM, Ellison EP, Venkatesh AK, Engel KG, Cameron KA, Makoul G, Adams JG: Emergency department team communication with the patient: the patient's perspective. J Emerg Med. 2013, 45(2):262-270.

28. Bücker JJ, Furrer O, Poutsma E, Buyens D: The impact of cultural intelligence on communication effectiveness, job satisfaction and anxiety for Chinese host country managers working for foreign multinationals. Int J Hum Resour Manage. 2014, 25(14):2068-2087.

29. Diallo AF, McGrath JM: A glance at the future of cultural competency in healthcare. Newborn and Infant Nurs Rev. 2013, 13(3):121-123.

30. Ezenkwele UA, Roodsari GS: Cultural competencies in emergency medicine: caring for Muslim-American patients from the Middle East. $J$ Emerg Med. 2013, 45(2):168-174.

31. Wesołowska K, Hietapakka L, Elovainio M, Aalto A-M, Kaihlanen A-M, Heponiemi T: The association between cross-cultural competence and well-being among registered native and foreign-born nurses in Finland. PloS one. 2018, 13(12):e0208761.

32. Crowne KA: Cultural exposure, emotional intelligence, and cultural intelligence: An exploratory study. Int J Cross Cult Manage. 2013, $13(1): 5-22$.

33. Suk MH, Oh W-O, Im Y: Factors affecting the cultural competence of visiting nurses for rural multicultural family support in South Korea. BMC nurs. 2018, 17(1):1.

34. AbuDagga A, Mara CA, Carle AC, Weech-Maldonado R: Factor Structure of the Cultural Competence Items in the National Home and Hospice Care Survey. Medical care. 2018, 56(4):e21-e25.

35. Ring J, Nyquist J, Mitchell S: Curriculum for culturally responsive health care: The step-by-step guide for cultural competence training: CRC Press; 2018. 36. Mechanic OJ, Dubosh NM, Rosen CL, Landry AM: Cultural competency training in emergency medicine. J Emerg Med. 2017, 53(3):391-396.

37. Kaihlanen A-M, Hietapakka L, Heponiemi T: Increasing cultural awareness: qualitative study of nurses' perceptions about cultural competence training. BMC nurs. 2019, 18(1):1-9. 